\title{
Educação Em Saúde: Cuidados Paliativos
}

\section{Health Education: Palliative Care}

\section{Resumo}

O presente texto apresenta a definição de Cuidados Paliativos, um breve histórico dos Cuidados Paliativos no mundo e no Brasil. Aponta a importância da Educação em Cuidados Paliativos por meio da educação, seja educação permanente, educação continuada ou educação em serviço. Além disso, indica a importância da incorporação do Ensino em Cuidados Paliativos nos currículos dos cursos de medicina e dos cursos de graduação na área da saúde, possibilitando proporcionar melhores recursos de cuidado na cura e na terminalidade de vida dos pacientes.

Palavras-Chave: Cuidados Paliativos; Educação; Educação Permanente; Educação Continuada; Formação Profissional.

\section{Abstract}

This text presents the definition of Palliative Care and a brief history of Palliative Care around the world and in Brazil. It highlights the importance of Palliative Care Education through different means, be it permanent, continuous, or in-service. In addition, it demonstrates the importance of que incorporation of palliative care teaching into the curricula of medical courses and graduate courses in the health sector, enabling the provision of better care resources for the treatment of the end-of-life stage of the patients.

Keywords: Palliative Care; Education; Permanent Education; Continuing Education; Professional qualification

\section{Introdução}

Ao pensarmos em cuidado paliativo, inicialmente temos no senso comum que paliativo possui uma conotação de "gambiarra" porque "não tem nada para fazer".

Na área da saúde, porém, segundo Carvalho (2015), a abordagem do Cuidado Paliativo tem como objetivo a prevenção e o alívio do sofrimento humano e precisa ser multiprofissional, enxergando a pessoa doente e sua família nas dimensões física, psíquica, social/ familiar e espiritual.
Prof. ${ }^{a}$ Dr. ${ }^{a}$. Rita Maria

Lino Tarcia

Doutora em Linguística: Semiótica e Linguística Geral pela Universidade de São Paulo; Professora Adjunta do DIS da UNIFESP, Coordenadora Pedagógica do Curso de Especialização em Saúde da Família da Universidade Aberta do SUS (UNA-SUS/UNIFESP), Docente e Orientadora credenciada do Programa de Mestrado Profissional de Ensino em Ciências da Saúde do Centro de Desenvolvimento Superior em Saúde (CEDESS/ UNIFESP); Diretora da Associação Brasileira de Ensino a Distância (ABED).

Email: rtarcia@uol.com.br

Prof. ${ }^{a}$ Ma. Ana Cláudia Arguelles dos Reis

Mestre em Ensino em Ciências da Saúde pela Universidade Federal de São Paulo, Especialista em Cuidados Paliativos pela Faculdade Santa Marcelina, Especialista em Trabalho Social com Famílias pelo Centro Universitário das Faculdades Metropolitanas Unidas, Assistente Social da oncologia e cuidados paliativos do Hospital Santa Marcelina Itaquera.

Email: anaclaureis@ hotmail.com 
O mesmo autor menciona que, no sentido etimológico, pallium significa aquele que protege e oculta os sintomas de uma doença em franca evolução. Historicamente, o termo estaria ligado ao manto dos cavaleiros das Cruzadas, que os protegeria das variações climáticas.

Outro conceito é hospice, que inicialmente significa hospedeiro e posteriormente estranho, hóspede, viajante. Outro sentido do termo hospice diz respeito à filosofia que embasa a prática de cuidados paliativos, hospice care, e o local destinado a esse cuidado realizado por uma equipe multiprofissional focada no paciente, em suas necessidades clínicas e resolução de questões pessoais de ordem: familiar, financeira, espiritual, entre outras.

A definição de Cuidados Paliativos em 1986, segundo a World Health Organization (WHO), é:

Cuidado ativo e total para pacientes cuja doença não é responsiva a tratamento de cura. Controle da dor, de outros sintomas e de problemas psicossociais e espirituais são primordiais. O objetivo do Cuidado Paliativo é proporcionar a melhor qualidade de vida possível para pacientes e familiares. (WHO, 1986, tradução nossa.)

Em 2002, segundo a World Health Organization, cuidados paliativos tratam-se da

\begin{abstract}
Abordagem que aprimora a qualidade de vida dos pacientes e famílias que enfrentam problemas associados com doenças ameaçadoras de vida, através da prevenção e alívio do sofrimento, por meio de identificação precoce, avaliação correta e tratamento da dor e outros problemas de ordem física, psicossocial e espiritual. (WHO, 2002, tradução nossa.)
\end{abstract}

Já em 2017, com o processo de aprimoramento do trabalho de cuidados paliativos, a definição passou a ser:

Cuidado Paliativo "uma abordagem que melhora a qualidade de vida dos pacientes (adultos ou crianças) e de seus familiares que enfrentam problemas associados a doenças que ameaçam a vida. Previne e alivia sofrimento por meio da investigação precoce, avaliação correta e tratamento da dor e de outros problemas 'físicos, psicossociais ou espirituais"'. (WHO, 2017, tradução nossa.)

Sendo assim, faz-se necessário ir além do tratamento de sintomas físicos e buscar desenvolver um olhar técnico para as questões psicossociais e espirituais do paciente.

A WHO 2002 define os princípios básicos que norteiam os Cuidados Paliativos, considerando o que se expõe a seguir.

A morte é vista como natural e esperada na evolução de uma doença ameaçadora da vida e no oferecimento de apoio para auxiliar o paciente a viver da melhor maneira possível até o dia de sua morte.

O conhecimento técnico e específico deve buscar o alívio da dor, seja ela física, social, familiar, emocional e espiritual.

Os Cuidados Paliativos seriam a ortotanásia que diz respeito à morte natural, morte como um ciclo, morte como parte da vida.

Para estes cuidados são necessários diversos saberes profissionais e a equipe multiprofissional irá avaliar e cuidar desse paciente olhando-o como um todo, em todas as suas necessidades. Usa-se uma abordagem em equipe para orientar as necessidades do paciente e de seus familiares, incluindo orientação sobre luto, se indicado.

Cabe ainda aos Cuidados Paliativos oferecer apoio à família durante o período de doença do paciente e posteriormente no momento de luto, dentro da realidade daquela família, compreendendo as relações paciente - familiares e condições que a família possui para cuidar do paciente.

Tais cuidados são aplicáveis inicialmente no curso da doença, em conjunção com outras terapias que pretendem prolongar a vida, tais 
como quimioterapia ou radioterapia, e incluem os exames necessários para melhor entender e administrar as complicações clínicas que causam sofrimento.

Os pacientes que recebem Cuidados $\mathrm{Pa}$ liativos desde o diagnóstico de uma doença ameaçadora de vida têm a possibilidade de viver com mais qualidade de vida, pois não são submetidos a intervenções agressivas, fúteis e podem morrer com dignidade.

Apresentaremos a seguir um breve histórico dos Cuidados Paliativos no mundo e no Brasil.

\section{Cuidados paliativos na atenção à saúde no Brasil}

Durante muito tempo, o termo Hospice foi usado para denominar a prática dos Cuidados Paliativos. Primeiramente, o termo Hospice foi usado para definir espécies de abrigos para os peregrinos e viajantes. O relato mais antigo é do século V, Hospício do Porto de Roma, destinado aos viajantes oriundos da Ásia, áfrica e do Leste (CORTES, 1988).

Segundo Maciel (2008), na Europa, no caminho de Santiago de Compostela, havia os Hospices Medievais que abrigavam peregrinos e doentes, que recebiam o cuidado leigo e caridoso. No século XVII, as instituições de caridade construíram abrigos para órfãos, pobres e doentes. Essa prática se propagou e no século XIX, essas instituições começaram a ter características de hospitais, com alas para doentes com tuberculose e câncer, sendo o atendiment essencialmente leigo e voltado para o cuidado espiritual e a tentativa de controle da dor.

Maciel (2008) menciona também que em Londres, St. Luke's Home, em meados do século $X X$, a assistente social, enfermeira e médica Cicely Saunders dedicou-se ao estudo do alívio da dor nos doentes em terminalidade de vida. Ela também publicou artigos fundamentais descrevendo as necessidades dos doentes, o conceito da dor total e os cuidados necessários no final da vida. Em 1967, com o início do Movimento Hospice Moderno, Cicely fundou em
Londres o St. Christhofer Hospice, onde além da assistência aos doentes, foram fornecidos estudos para ensino e pesquisa.

Segundo a mesma autora, na década de 1970, o Movimento Hospice cresceu nos Estados Unidos, com o encontro de Cicely Saunders e Elizabeth Klüber-Ross (psiquiatra norte-americana). Em 1975, foi fundado em Connecticut o primeiro Hospice Americano. E em 1982, surge o Hospice Care, promovendo ações especialmente de cuidado domiciliar, através de um sistema de reembolso. Neste mesmo ano, - Comitê de Câncer da Organização Mundial de Saúde criou um grupo de trabalho para definir políticas que atendessem os doentes com câncer, do tipo Hospice. Devido a dificuldades de tradução fidedigna do termo Hospice em alguns idiomas, passou-se a utilizar o termo Cuidados Paliativos, este termo já havia sido usado no Canadá em 1975.

Maciel (2008) menciona a publicação do estudo multicêntrico realizado entre 1989 e 1994 envolvendo cerca de dez mil pacientes paliativos com o prognóstico de vida estimado em seis meses, que apontou questões fundamentais no final da vida: a comunicação entre pacientes e familiares com a equipe de saúde sobre o final da vida é pobre; o custo da atenção no final da vida é elevado e metade dos pacientes morre com dor moderada ou severa, sem nenhuma prescrição analgésica (SUPPORT, 1995).

O modelo de cuidados paliativos chegou ao Brasil no início da década de 1980, durante o regime de ditadura, com o sistema de saúde priorizando a modalidade hospitalocêntrica, essencialmente curativa. Nossos médicos, psicólogos e teólogos foram para o Exterior para conhecer a realidade dos cuidados paliativos de outros países para adaptarem a filosofia hospice à realidade brasileira e passaram a difundir o conhecimento em Cuidados Paliativos. Com isso, houve um avanço dos Cuidados Paliativos no país e em 1997 foi fundada a Associação Brasileira de Cuidados Paliativos (ABCP). Em 2005, foi criada a Associação Nacional de Cuidados Paliativos (ANCP), que impulsionou a discussão sobre os cuidados paliativos e a luta 
por sua inserção nos programas de atenção à saúde, assim como para o reconhecimento da Medicina Paliativa como especialidade médica (RODRIGUES; ZAGO, 2010)

Segundo Maciel (2008), em 2002, a OMS publica os estudos The Solid Facts of Palliative Care e Better Care of the Elderly e ambos recomendaram os Cuidados Paliativos como estratégia de ação em sistemas nacionais de saúde. Os Cuidados Paliativos passam a ampliar as áreas de conhecimento, incluindo a pediatria, geriatria, HIV/AIDS, doenças crônicas, entre outras.

Segundo Andrade (2015), no Brasil, embora pouco conhecidos e divulgados, os Serviços de Cuidados Paliativos são realizados em ambulatórios, enfermarias, nos domicílios e em hospices ou casas de cuidado, sendo que o atendimento em ambulatório destina-se a pacientes que ainda possuem condições clínicas e suporte social que lhes permitem a ida ao ambulatório.

Teixeira e Lavor (apud Andrade, 2015) mencionam que o serviço mais antigo registrado em nosso país é o realizado pelo Instituto Nacional de Câncer (INCA), no Rio de Janeiro, desde 1986, oferecendo o primeiro projeto de atenção domiciliar para pacientes com câncer e fora de possibilidades terapêuticas (FPT), que ao ser desdobrado incluiu a modalidade de assistência ambulatorial. O serviço é composto por uma equipe formada por médicos, enfermeiros, assistentes sociais, nutricionistas, psicólogos e fisioterapeutas.

Andrade (2015) menciona que esse serviço realiza o atendimento emergencial 24 horas por dia. O referido ambulatório oferece ainda a assistência a distância mediante contato telefônico, para esclarecimentos de dúvidas e orientando situações de algumas intercorrências simples, evitando à ida do paciente ao Hospital.

Andrade (2015) também cita que em março de 1995 foi criado o Ambulatório de Cuidados Paliativos do Hospital das Clínicas da Faculdade de Medicina da USP (ACP/HMUSP) para atender os pacientes encaminhados das diversas clínicas que compõem o complexo do Hospital das Clínicas, sendo, na ocasião, a equipe composta por médico, enfermeira, assistente social, psicólogo e terapeuta ocupacional voluntário e atualmente, por médicos, assistentes sociais, farmacêuticos e assistentes espirituais. Além disso, o atendimento conta também com a internação hospitalar em casos mais graves.

Maciel (2008) menciona que em 2002, em São Paulo, foi criada a enfermaria de Cuidados Paliativos do Hospital do Servidor Público Estadual Francisco Morato de Oliveira (HSPE-SP), estendida ao atendimento ambulatorial e domiciliar. A equipe era constituída por médicos, enfermeiras, psicólogo, assistente social, assistentes espirituais (voluntários). Além destes, fisioterapeutas, odontólogos, nutricionistas e especialistas de outras áreas do hospital podem ser chamados para intervenções específicas.

Andrade (2015) menciona que a alternativa hoje existente entre a internação em enfermarias e a assistência domiciliar, em se tratando de cuidados paliativos, é o hospice ou casa de cuidados. Atualmente, temos registro de alguns serviços estruturados em São Paulo, são eles:

Pavilhão Anna Cândida de Carvalho - Centro do Hospital Amaral Carvalho (HAC), em Jaú: primeiro serviço estruturado no Brasil, fundado em 1993, com nove leitos.

Casa de Apoio-Hospedaria para Cuidados Especiais, pertencente ao Hospital do Servidor Público Municipal de São Paulo: criada em julho de 2004, sob coordenação da Dra. Dalva Yukie Matsumoto, com dez leitos, referência em hospice devido à qualidade do serviço prestado, ao trabalho em equipe e ao modelo de atenção focado no cuidado ao paciente que está em terminalidade de vida e na aceitação da morte como parte da vida. Oferece cuidados aos pacientes sem famílias ou com dificuldades de serem assistidos em seus respectivos domicílios.

Hospice do HCFMUSP, ligado ao Instituto do Câncer de São Paulo: suas ações iniciaram-se em 1995. Em 2008, houve a implantação de uma comissão de cuidados paliativos com o objetivo de estruturar essa modalidade de assistência 
em todo o complexo HC. Em 2011, foi aprovado o regimento interno da Comissão de Cuidados $\mathrm{Pa}$ liativos da Diretoria Clínica do HCFMUSP, abrangendo a assistência de ambulatório, interconsulta, enfermaria, assistência domiciliar e hospice.

Ainda conforme Andrade (2015), dados da Associação Brasileira de Cuidados Paliativos (ABCP) estimam que existam cerca de 30 núcleos que exercem serviços de atendimento à dor e cuidados paliativos, sendo 16 no Estado de São Paulo, entre eles: Santa Casa de São Paulo, Hospital Pérola Byington, Hospital das Clínicas da FMUSP, Hospital AC Camargo, IC Arnaldo Vieira de Carvalho, Hospital Emílio Ribas, Hospital do Servidor Público, Hospital Heliópolis.

O Serviço de Cuidados Paliativos no Hospital Santa Marcelina iniciou-se em 2006 com o atendimento de 01 médico na Retaguarda do Pronto-Socorro. Em 2007, 01 médico e uma enfermeira iniciaram as interconsultas. No mesmo ano, a equipe passou a ser constituída por médico, enfermeiro, psicólogo, assistente social, farmacêutico, fisioterapeuta, nutricionista e conselheiro espiritual. Em 2008, foram implantados o ambulatório e a enfermaria. Inicialmente, o ambulatório realizava atendimentos às terças-feiras no período da tarde. Na atualidade, o ambulatório realiza os atendimentos às segundas, terças e sextas-feiras no período da tarde. Atualmente, a equipe multiprofissional é composta por médicos, enfermeiro, psicólogo, assistente social, fisioterapeuta, fonoaudiólogo e nutricionista.

Percebemos que os Cuidados Paliativos encontram-se em crescimento no Brasil, porém necessitam de sua constante organização e divulgação nos serviços hospitalares para que mais pacientes possam ser beneficiados por essa abordagem. Além disso, é imprescindível a busca pela educação em Cuidados Paliativos e a incorporação do ensino em Cuidados $\mathrm{Pa}-$ liativos nos currículos dos cursos de medicina e dos cursos de graduação na área da saúde, possibilitando proporcionar melhores recursos de cuidado na cura e na terminalidade de vida dos pacientes.

\section{Educação em saúde}

No processo educativo, é de suma importância trazer alguns conceitos sobre a educação. Paulo Freire (apud Gadotti, 1991) aprendeu a pensar sempre na prática. Segundo ele: "Estudar é realmente um trabalho difícil. Exige de quem o faz uma postura crítica sistemática. Exige uma disciplina intelectual que não se ganha a não ser praticando'".

Gadotti (1991) relata que para Paulo Freire estudar é uma forma de reivindicar, de recriar, de reescrever - tarefa de sujeito e não de objeto. Menciona ainda que o ciclo do conhecimento apresenta-se em dois momentos: momento da produção de conhecimento de algo novo e momento em que o conhecimento produzido é conhecido ou percebido. Para Paulo Freire, a forma de trabalhar o processo do ato de aprender era determinante em relação ao próprio conteúdo da aprendizagem, ou seja, é com a prática que efetivamente se aprende.

Gadotti (1991) menciona que métodos novos em que professores e alunos aprendem juntos são mais eficazes, desta forma o aprendizado é estímulo para ambos. A proposta de alfabetização, para Paulo Freire, surgiu como uma consequência natural da tomada de consciência dos problemas vividos pelo grupo que estava sendo alfabetizado. Este demonstrou maior interesse na aprendizagem por se identificar com as questões trazidas a partir do concreto vivido, no processo de aprendizagem. Sendo assim, aprender faz parte do ato de se libertar, de se humanizar.

Para Paulo Freire (apud Gadotti, 1991), conhecer é um processo social, e o diálogo é justamente o cimento desse processo. $O$ diálogo faz parte dessa nova pedagogia dialógico- dialética. O método dialético de pensar não separa teoria e prática. Teoria, método e prática formam um todo, guiado pelo princípio da

1 Extraído de um texto escrito por Paulo Freire, em 1968, no Chile, para um seminário sobre educação e reforma agrária (GADOTTI, p. 28, 1991). 
relação entre o conhecimento e o conhecedor. Na teoria dialógica, a colaboração, a união e a organização são essenciais, nela o educador libertador estimula os educandos a pensar. A educação é um momento do processo de transformação do homem disseminado pela transformação do mundo.

Nos Cuidados Paliativos, o processo de cuidado também parte do concreto vivido e o diálogo é um instrumento utilizado para conseguir melhores resultados, além de ser indispensável o diálogo na equipe. Precisamos perceber se a equipe conversa, pois é nessa relação por meio do diálogo em equipe que a educação deve acontecer.

Paulo Freire (apud Gadotti, 1991) discute a sua teoria do conhecimento baseando-se em quatro 04 dimensões: a lógica, relacionada aos métodos do conhecimento; a histórica, referente à relação entre conhecimento e contexto; a gnosiológica, referente às finalidades do conhecimento; e a dialógica, referente à comunicação do conhecimento. $O$ sujeito não pensa sozinho, a comunicação deve ser simples mesmo quando o assunto é complexo e trazer essa comunicação para o concreto vivido possibilita a simplicidade, indo-se a fundo no assunto de maneira acessível e não complicada.

Na área da saúde, é fundamental para a realização de um trabalho adequado que a equipe multiprofissional busque constantemente conhecimentos individualmente e/ou em grupo. Esses conhecimentos em grupo se realizam por meio da educação permanente, educação continuada ou educação em serviço. Definiremos estes conceitos a seguir.

Segundo Paschoal et al. (2007), a educação é um fenômeno social e universal, que precisa cuidar da formação de seus indivíduos, fornecendo subsídios para a participação ativa e transformadora na sua vida social. É também o processo para fornecer os sujeitos do conhecimento e das experiências culturais, entre outras que os tornam capacitados a atuar no meio social. $O$ homem precisa de uma busca contínua como um ser ativo na construção do seu saber, sendo o responsável por sua educação e procurando recursos que o levem ao crescimento e aperfeiçoamento de sua capacidade. Portanto, a educação é um processo dinâmico e contínuo de construção do conhecimento, que leva a uma consciência crítico-reflexiva e, consequentemente, à criação de compromisso pessoal e profissional, capacitando-o para a transformação da realidade.

Peduzzi et al. (2009) mencionam a implantação da educação permanente em saúde (EPS) como política nacional para formação e desenvolvimento de trabalhadores da saúde, sendo que esta política pública propõe que os processos de capacitação dos trabalhadores surgiram a partir das necessidades de saúde das pessoas e das populações, da gestão setorial e do controle social em saúde.

Os autores citam que a Portaria 1996/07 estabeleceu novas diretrizes e estratégias para a implementação dessa política, de modo a adequá-la às diretrizes operacionais e ao regulamento do Pacto pela Saúde, que define a política de recursos humanos para o Sistema Único de Saúde (SUS) como eixo estruturante que deve buscar a valorização do trabalho e dos trabalhadores da saúde (Brasil, 2007).

Segundo os autores, no início dos anos 80, a Organização Pan-Americana da Saúde lançou a proposta da EPS, com a finalidade de reconceituar e reorientar os processos de capacitação de trabalhadores dos serviços de saúde, tendo como eixo da aprendizagem o trabalho executado no cotidiano dos serviços, como processo permanente de natureza participativa e multiprofissional. A EPS compreendida como espaço de problematização, reflexão, diálogo e construção que possibilita promover mudanças e transformações na perspectiva da integralidade da saúde. A EPS parte da concepção de educação como transformação e aprendizagem significativa baseada no exercício cotidiano do processo de trabalho e de que as práticas 
são definidas por fatores voltados à multiprofissionalidade e à interdisciplinaridade, com estratégias de ensino participativas e focadas na transformação das práticas.

Segundo Paschoal et al. (2007), a questão educativa no contexto da prática e do desenvolvimento profissional pode ser percebida como educação permanente em saúde (EPS), educação continuada (EC) e educação em serviço.

Os autores mencionam que a EPS surge como uma exigência na formação do sujeito e baseia-se no aprendizado contínuo para o desenvolvimento do sujeito direcionado à busca da competência pessoal, profissional e social, como meta constante. Consiste no desenvolvimento pessoal que deve ser potencializado, promovendo a capacitação técnica, a aquisição de novos conhecimentos, conceitos e atitudes.

Segundo os mesmos autores, a EC seria toda a ação desenvolvida após a profissionalização com a finalidade de atualizar conhecimentos e adquirir novas informações e atividades de duração por meio de metodologias formais. É conceituada como um conjunto de experiências adquiridas após a formação inicial, permitindo ao trabalhador manter, aumentar ou melhorar sua competência, tornando-a compatível com o desenvolvimento de suas responsabilidades. É um conjunto de práticas educativas contínuas, objetivando o desenvolvimento de potencialidades para a transformação de sua prática.

Para Peduzzi et al. (2009), a EC parte da concepção de educação como transmissão de conhecimento e pela valorização da ciência como fonte do conhecimento; é pontual, fragmentada e construída de forma não articulada à gestão e ao controle social, focada nas categorias profissionais e nos conhecimentos técnicos científicos de cada área, com cursos e treinamentos baseados no diagnóstico de necessidades individuais, com a perspectiva de transformação da organização em que o profissional está inserido.

Segundo Paschoal et al. (2007), a educação em serviço constitui-se em um processo educativo aplicado nas relações humanas do trabalho, no intuito de desenvolver capacidades cognitivas, psicomotoras e relacionais dos profissionais, além de aperfeiçoamento diante da evolução científica e tecnológica. Seu desenvolvimento processa-se no ambiente de trabalho de uma instituição em particular. Nela, destacam-se quatro áreas de atuação: a orientação ou introdução ao trabalho; treinamento; atualização; e aperfeiçoamento, aprimoramento ou desenvolvimento. Na educação em serviço, os projetos de ensino devem atender às necessidades daqueles que deles vão participar e aos objetivos da instituição.

Segundo os mesmos autores, a EPS, EC e a educação em serviço se fundamentam em diferentes princípios metodológicos e têm caráter de continuidade do processo educativo. Entendem que a educação permanente é mais ampla, fundamentando-se na formação do sujeito, enquanto a educação continuada e a em serviço estão contidas na permanente.

O trabalho realizado pela equipe multiprofissional em Cuidados Paliativos necessita da EPS e corrobora com Peduzzi et al. (2009) e com Paulo Freire (apud Gadotti, 1991), pois a partir das necessidades da equipe, elaboramos um processo de reflexão, diálogo e construção na busca por mudanças na perspectiva de aprimorar o atendimento prestado aos pacientes e seus familiares.

\section{Cuidados paliativos e a formação profissional em saúde no Brasil}

Atualmente, a população brasileira vive mais tempo, porém convivendo com doenças crônicas com considerável sofrimento para o paciente e a família. Diante deste contexto, faz-se necessário e urgente instrumentalizar profissionais para atuarem em CP.

Figueiredo e Stano (2013) mencionam que na Inglaterra, o primeiro país em que a Medicina Paliativa passou a ser considerada como especialidade médica, o Dr. Derek Doyle, autoridade internacional em Cuidados Paliativos, afirmou 
que o objetivo era apenas que os médicos se interessassem em exercê-la e para que os outros especialistas os respeitassem, não havendo motivação pelo paciente e necessidade de cuidado integral.

Não desejamos que os Cuidados Paliativos sejam vistos pelo olhar do Dr. Derek, como citado. Mas pela visão de Figueiredo e Stano (2013), que reforçam a importância de preparar ofuturo médico desde o início de sua formação para que ele compreenda a necessidade de melhor sintonia entre a técnica e a proximidade empática e afetiva com o paciente, entendendo a importância da família como parte da vida do paciente e parte fundamental no processo de cuidado, para que ele saiba contribuir com a interação entre os diversos saberes profissionais de uma equipe de cuidados e para que saiba respeitar e estimular a necessidade de apoio espiritual dos pacientes e familiares.

Lampert et al. (2009) menciona que no Brasil a legislação quanto ao sistema nacional de saúde tem avanços relevantes para mudanças na prestação de serviços na área da saúde. A partir da Constituição Brasileira de 1988, as leis complementares e as normas operacionais básicas têm possibilitado a evolução na estruturação deste sistema. Neste contexto, são homologadas as Diretrizes Curriculares Nacionais (DCN) para os cursos de graduação pelo Conselho Nacional de Educação do Ministério da Educação, que apontam as características do profissional da área da saúde a ser formado: generalista, humanista, crítico e reflexivo, capaz de trabalhar em equipe, para que as escolas adaptem seus projetos político-pedagógicos para formar profissionais com competência para atender às necessidades de saúde na dinâmica do contexto, e que o SUS deve regular a formação de recursos humanos para o atendimento em saúde. Para avaliar essas ações, foi criado o Sistema Nacional de Avaliação da Educação Superior (Sinaes).

Segundo Lampert et al. (2009), as DCN delineiam o perfil que o médico deve alcançar ao concluir o curso de graduação, com o objetivo de adquirir competências para trabalhar em equipe, dar conta de atender com qualidade às necessidades mais frequentes de saúde e saber construir o conhecimento a partir da formulação de problemas encontrados no dia a dia do exercício profissional e dar soluções adequadas. Orientam as escolas para inserir em seus currículos conteúdos essenciais relacionados ao processo saúde-doença do cidadão, da família e da comunidade, integrados à realidade epidemiológica e profissional, proporcionando a integralidade das ações do cuidar em medicina. Além disso, devem contemplar a compreensão dos determinantes sociais, culturais, comportamentais, psicológicos, éticos e legais, nos níveis do indivíduo e do coletivo, no processo saúde-doença.

Para Lampert et al. (2009), de acordo com sua vocação institucional, a Abem (Associação Brasileira de Educação Médica) propõe, por intermédio da Caem (Comissão de avaliação de Educação Médica), promover a avaliação das tendências de mudanças dos cursos de graduação das escolas médicas brasileiras, tendo por objetivos impulsionar e auxiliar a construção de um processo avaliativo nessas instituições. Desde março de 2006, a proposta de avaliação da Caem, além de diagnosticar o momento das escolas, visa auxiliar e acompanhar a evolução das mudanças de forma participativa e construtiva para atender às DCN com perspectivas à consolidação do SUS, incentivando a construção do processo avaliativo em cada escola médica dentro dos princípios do Sinaes, com vista à formação do médico no curso de graduação.

Saito e Zoboli (2015) afirmam que atualmente ocorre um aumento das doenças crônicas que possuem uma evolução progressiva e degenerativa e essas condições crônicas de saúde geram uma demanda por assistência contínua e permanente, incluindo os Cuidados Paliativos (CP).

Segundo Figueiredo e Stano (2013), as Escolas de Medicina beneficiariam a formação dos futuros médicos se incorporassem aos seus currículos o ensino de Cuidados Paliativos, possibilitando complementar o aprendizado da 
medicina e proporcionando melhores recursos de cuidado na cura e na terminalidade de vida.

De acordo com as autoras:

Define-se por currículo a tessitura de informações, práticas, compartilhamento de conceitos e reflexões conjuntas professor-aluno, que propiciam a ambos a construção ativa do conhecimento necessário ao exercício da vida profissional do aluno e ao contínuo aprimoramento do saber do professor. A função socializadora do currículo ultrapassa o seu valor como apenas transmissão de conteúdo. Assim, o conceito por trás da composição dos conteúdos do currículo não se dissocia dos conceitos e valores desejáveis pela sociedade à qual a escola pertence. Pode-se até dizer que toda escola se esforça por propiciar o mais próximo do "ideal de homem" que a sociedade elegeu na época considerada, sendo hoje notória a demanda pela formação de sujeitos de direitos, dignos e verdadeiramente incluídos na trama social. (FIGUEIREDO \& STANO, 2013, p. 299.)

As autoras referem que a partir de $1^{\circ}$ de agosto de 2010, por conta da Resolução CFM $n^{\circ} 1.973 / 2011$, a medicina paliativa tornou-se área de atuação de seis especialidades médicas (geriatria, pediatria, oncologia, clínica médica, anestesiologia, medicina da família). Hoje já são oito as especialidades médicas. Às seis anteriores somaram-se Medicina Intensiva e Cirurgia de Cabeça e Pescoço (Resolução CFM 2068/2013).

No I Encontro Latino-Americano de Docentes de Cuidados Paliativos em Buenos Aires para divulgação entre os países da América Latina, a orientação sugerida pela International Association for Hospice and Palliative Care (IAHPC) recomenda que, no mundo inteiro, se procure sensibilizar os governos, as instituições de ensino e as associações de classe para que a educação em CP se inicie no primeiro ano da graduação das várias profissões da saúde. A delegação brasileira era uma das maiores presentes. Esta recomendação baseou-se na experiência europeia dos últimos anos. Na Europa, após anos de educação em CP em cursos de pós-graduação, inicialmente pensados como forma de capacitar mais rapidamente os profissionais já formados, verificou-se a necessidade de iniciar a sensibilização dos profissionais no decorrer da graduação, o que facilitaria a construção de competências adequadas à prática dos CP. Esse talvez seja o caminho no Brasil, o de proporcionar a todos os médicos o contato com a medicina paliativa na esperança de responder à crescente demanda de cuidados integrais a pacientes crônicos e à sua família.

Figueiredo e Stano (2013) mencionam que no Brasil, no período de 1994 a 2008, a Universidade Federal de São Paulo (Unifesp), por meio do Prof. Marco Tullio Barcellos de Assis Figueiredo (1925-2013), foi a primeira escola médica a disponibilizar os cursos de CP em caráter eletivo a alunos de graduação em medicina, enfermagem e fisioterapia. O Prof. Marco Tullio, falecido em 2013, foi considerado o pioneiro em educação em CP no Brasil e iniciou o binômio cuidado paliativo e formação profissional. Foi Professor da disciplina de Cuidados Paliativos da UNIFESP-EPM, Chefe do Ambulatório de Cuidados Paliativos da UNIFESP-EPM, Sócio fundador da International Association for Hospice and Palliative Care (USA) São Paulo, SP.

Em 1994, procurou a diretoria do Departamento Científico e Cultural (DCC) do Centro Acadêmico Pereira Barreto da UNIFESP-EPM, com o projeto de montar um "Curso de Cuidados Paliativos ao Paciente fora de Recursos Terapêuticos de Cura". E o projeto foi adiante. Na primeira quinzena do mês de novembro de 1994, ocorreu o $1^{\circ}$ curso, que continuou a ser realizado. Em 1997, foram incluídos cursos sobre Tanatologia - Estudo da Morte como sequência ao curso de Cuidados Paliativos e a disciplina de Oncologia Básica. Com isso, a Pró-Reitoria de Extensão de Graduação conferiu a estes dois cursos o título de "Cursos de Extensão".

Com o decorrer dos anos, o curso de Cuidados Paliativos e Tanatologia possibilitaram a criação da disciplina eletiva de Cuidados 
Paliativos em 1998 e a formação do Ambulatório de Cuidados Paliativos em 2000. Em junho de 2000, o Dr. Marco Tullio submeteu à apreciação do Reitor Prof. Dr. Hélio Egídio Nogueira um dossiê sobre o programa de educação em Cuidados Paliativos executado na UNIFESP-EPM desde 1994. No dossiê, ele sugeriu ao Reitor a necessidade de organizar um ambulatório de Cuidados Paliativos para atender ao crescente número de portadores de câncer avançado atendidos na oncologia do Hospital São Paulo, iniciar um programa de formação e treinamento de profissionais em Cuidados Paliativos para atender os pacientes do Hospital São Paulo (HSP) e UNIFESP-EPM e também para atuar em outros hospitais, oferecer estágios aos residentes e alunos da universidade.

Em dezembro de 2000, conseguiu iniciar o atendimento ambulatorial com uma equipe formada por um médico, uma enfermeira efetiva e uma R2, duas psicólogas, uma assistente social e um dentista. A unidade não dispunha de leito fixo no HSP. O atendimento básico era ambulatorial e domiciliar. No caso de o paciente não ter mais condições físicas de locomoção ao Ambulatório, eram agendadas visitas domiciliares.

Em 2002, a UNIFESP-EPM contava com a atividade didática em Cuidados Paliativos e Tanatologia, alcançando uma posição de destaque internacional. Com um total de 41 horas/ ano de cursos de informação, a UNIFESP-EPM ocupava o primeiro lugar entre as universidades do mundo. Ela era a única que estendeu à comunidade a oportunidade de participar destes cursos (cursos de DCC).

Em 2003, participou da criação da disciplina de CP e do Serviço de CP na Faculdade de Caxias do Sul - RS, que se iniciaram em 2004 e continuam em funcionamento.

Em 2004, participou das reuniões que deram origem à Academia Nacional de Cuidados Paliativos (ANCP), tornando-se membro honorário desta entidade. Foi também sócio-fundador da International Association for Hospice and Palliative Care (IAHPC, 1997) e membro de seu conselho consultivo durante três mandatos. Em 2008 participou da elaboração do livro Cuidado Paliativo, do Conselho Regional de Medicina de São Paulo (Cremesp), juntamente com sua esposa, a psiquiatra paliativista Dra. Maria das Graças Mota Cruz de Assis Figueiredo.

O Prof Marco Tullio Barcellos de Assis Figueiredo deixou o seu legado na formação profissional, que incentivou outras ações no decorrer dos anos.

Figueiredo e Stano (2013) acompanharam o esforço de um doutorando da Universidade Federal de Minas Gerais que desejava investigar o número de Faculdades de Medicina do país que disponibilizam disciplinas de Cuidados Paliativos aos seus alunos, considerando que temos mais de 180 Escolas de Medicina no país. Sua amostra foi reduzida apenas às Faculdades de Minas Gerais, constando que apenas na Universidade José do Rosário Vellano - Unifenas (método $\mathrm{ABP}$ ), no $4^{\circ}$ período, com duração de um semestre, existe a disciplina de Estratégia de Prática Médica na Comunidade, com foco em Terminalidade e Cuidados Paliativos. E também a Faculdade de Medicina de Itajubá (FMIt) oferece em caráter curricular aos alunos de primeiro, segundo e quarto anos da graduação a disciplina de Tanatologia e Cuidados Paliativos (TanCP) desde 2010, sendo que no primeiro e segundo anos, a disciplina oferece $36 \mathrm{~h}$ e no quarto ano, $18 \mathrm{~h}$, a grupos de 40 alunos a cada 15 dias. Vale ressaltar que a disciplina teve que se ajustar à carga horária disponível e não à necessidade real de tempo para o aprendizado necessário referente à prática dos conceitos.

A criação dessa disciplina se deve ao grande interesse pelo assunto que tinha o Dr. Kleber Lincoln Gomes, presidente da Entidade Mantenedora da FMIt (AISI), e da Dra. Christina Grieger, vice-diretora da Faculdade (falecida em janeiro de 2010). Eles entraram em contato com CP através de um Hospital em São Paulo dirigido por um ex-aluno da FMIt, que implantou as práticas de CP com a assessoria do Prof. Marco Tullio. Nesta disciplina, sempre foi enfatizada 
aos alunos a oportunidade única de auxiliarem a outros na obtenção do conhecimento em si e do conhecimento sobre CP. Em início de 2011, esta disciplina criou um projeto extracurricular chamado A Arte do Cuidar, com o objetivo de elaborar material didático produzido por alunos e destinado a ensinar outros, já tendo elaborado 04 cursos de CP; coordenado estágios de observação em CP para cerca de 120 alunos, hoje cerca de 500 alunos em serviços parceiros nas cidades de São Paulo, Barretos e Rio de Janeiro; organizado dois saraus de CP, atualmente 05 saraus; e publicado dois manuais de CP, três vídeos educativos e também um gibi chamado Tanatoons, que fala de perdas e morte para crianças.

Figueiredo e Stano (2013) concluem que todos os médicos devem ser capazes de assistir os seus pacientes até a morte, aliviando os sintomas e podendo oferecer conforto aos pacientes e seus familiares. Assim, aos especialistas em CP caberia assistir os pacientes que necessitem de técnicas mais específicas e que exijam maior prática dos seus médicos, semelhante ao que deve ocorrer entre um clínico e um especialista. Cabe às escolas de medicina responder a esta proposta de instrumentar todos os médicos desde o início de sua formação a aceitarem que a morte faz parte do ciclo maior da existência e que cuidar dos pacientes em terminalidade de vida faz parte da nobre missão médica. Para isso, fazem-se necessárias mudanças na grade curricular, com inclusão de novas disciplinas centradas no cuidado integral ao paciente e família.

Experiências como o projeto extracurricular A Arte do Cuidar precisam ser vivenciadas por diversas Escolas de Medicina, pois contribuirão na formação profissional especializada, para que pacientes, familiares e toda a sociedade possam usufruir desses benefícios.

No Hospital Santa Marcelina, acompanhamos os 02 residentes médicos- $\mathrm{RO1}$, que permanecem 30 dias nas enfermarias de Geriatria- $3^{\circ} \mathrm{D}$ e Cuidados Paliativos- $3^{\circ}$ D. Além destes, acompanhamos os residentes da Oncologia Hematológica, cuja residência foi credenciada em 19 de dezembro de 2013 pela CNRMS/MEC e possui equipe composta por 01 enfermeira, 01 nutricionista, 01 fisioterapeuta e 01 psicólogo, que realizam a residência no período de 02 anos, distribuídos da seguinte forma: no período de 06 meses permanecem na Oncologia Clínica e Ambulatório de Quimioterapia, 06 meses na Oncologia Cirúrgica, 06 meses na Hematologia e Transplante de Medula Óssea (TMO) e 06 meses na Enfermaria de Cuidados Paliativos.

Os residentes médicos e os residentes da Oncologia Hematológica são supervisionados pela equipe médica e multiprofissional, além de participarem das reuniões semanais da Equipe Multiprofissional para discussão dos casos de pacientes internados e de aulas semanais específicas sobre as práticas dos Cuidados Paliativos.

\section{Considerações finais}

A abordagem do Cuidado Paliativo tem como objetivos a prevenção e o alívio do sofrimento humano e precisa ser multiprofissional, enxergando a pessoa doente e sua família nas dimensões física, psíquica, social/familiar e espiritual.

A morte é vista como natural e esperada na evolução de uma doença ameaçadora da vida e no oferecimento de apoio para auxiliar o paciente a viver da melhor maneira possível até o dia de sua morte (WHO, 2002).

O conhecimento técnico e específico deve buscar o alívio da dor, seja ela física, social, familiar, emocional e espiritual. Esse conhecimento deve ser adquirido por meio da educação, seja esta permanente, continuada ou educação em serviço. Porém, a Educação em Cuidados Paliativos deve ser iniciada muito antes, já no início da graduação de médicos e demais profissionais da área da saúde.

Precisamos com urgência de definições sobre a inclusão dos Cuidados Paliativos nas grades curriculares não só dos médicos, mas também dos profissionais de saúde, para que iniciem sua aproximação com os CP, e posteriormente dos Cursos de Educação em Cuidados Paliativos, 
que deverão possibilitar um conhecimento mais aprofundado aos profissionais, que, conjuntamente com a atuação prática, terão embasamento para uma melhor prática profissional, com o objetivo de possibilitar o alívio do sofrimento do paciente e oferecer o cuidado na fase de terminalidade de sua vida, porém o tempo de atuação na área será fundamental para uma melhor abordagem em CP e isso só se concretizará em médio a longo prazo.

\section{Referências}

ANDRADE, L. Cuidados paliativos: história. In: ANDRADE, L. (Org.). Cuidados paliativos e serviço social: um exercício de coragem. Holambra: Editora Setembro, 2015, p. 39-68.

BRASIL. Ministério da Saúde. Portaria n 1.996, de 20 de agosto de 2007. Disponível em: <http:// bvsms.saude.gov.br/bvs/saudelegis/gm/2007/ prt1996_20_08_2007.html>.Acesso em: 19 jun. 2018.

CARVALHO, R. T. de. Cuidados paliativos: conceitos. In: ANDRADE, L. (Org.). Cuidados paliativos e serviço social: um exercício de coragem. Holambra: Editora Setembro, 2015, p. 23-37.

CONSELHO FEDERAL DE MEDICINA. Resolução CFM No 1.973/2011. Disponível em: <https://portal. cfm.org.br/index.php?option=com_content\& view $=$ article\&id $=21971$ : conselho-federal - de medicina-cria-novas-areas-de-atuacaomedica\&catid=3>. Acesso em: 29 ago. 2018.

CONSELHO FEDERAL DE MEDICINA. Resolução CFM No 2068/2013. Disponível em: <http://www.portalmedico.org.br/resolucoes/ cfm/2013/2068_2013.pdf>. Acesso em: 29 ago./2018.

CORTES, C. C. Historia y desarrollo de los cuidados paliativos. In: Marcos G. S. Cuidados paliativos e intervención psicosocial en enfermos de cáncer. Las Palmas: ICEPS, 1988.

FIGUEIREDO, M. G. M.C. A; STANO, R. C. M. O estudo da morte e dos cuidados paliativos. Revista Brasileira de Educação Médica, v. 37, n. 2, 298-306, 2013. Disponível em: <http://www. scielo.br/pdf/rbem/v37n2/19.pdf>. Acesso em: 11 nov. 2017.

\section{GADOTTI, M. Convite à leitura de Paulo Freire.} Série Pensamento e Ação no Magistério. $2^{a}$ ed. São Paulo: Editora Scipione, 1991.

LAMPERT, J. B. et al. Tendências de mudanças em um grupo de escolas médicas brasileiras. Revista Brasileira de Educação Médica, v. 33, p. 19-34, 2009. Disponível em: <http://www.famema. $\mathrm{br} / \mathrm{ensino} / \mathrm{pdd} / \mathrm{docs} /$ tendencias.pdf $>$. Acesso em: 25 mar. 2018.

MACIEL, M. G. S. Definições e princípios. São Paulo: CREMESP, 2008.

PASCHOAL, A. S.; MANTOVANI, M. F.; MÉIER, M. J. Percepção da educação permanente, continuada e em serviço para enfermeiros de um hospital de ensino. Revista da Escola de Enfermagem da USP, São Paulo, v. 41, n. 3, p. 478-484, 2007. Disponivel em: <http://www.ee.usp. $\mathrm{br} / \mathrm{reeusp} / \mathrm{upload} / \mathrm{pdf} / 742 . p d f>$. Acesso em: 29 abr. 2017.

PEDUZZI, M. et al. Atividades educativas de trabalhadores na atenção primária: concepções de educação permanente e de educação continuada em saúde presentes no cotidiano de Unidades Básicas de Saúde em São Paulo. Interface - Comunic., Saúde, Educ., v. 13, n. 30, p. 121-34, jul./set. 2009. Disponível em: <http:// www.scielo.br/pdf/icse/v13n30/v13n30a11.pdf>. Acesso em: 29 abr. 2017.

RODRIGUES, I. G.; ZAGO, M. M. F. Cuidados paliativos: realidade ou utopia? Ciência, Cuidado e Saúde, Londrina, v. 8, p. 136-141, 23 abr. 2010. Disponível em: <http://periodicos. uem.br/ojs/index.php/CiencCuidSaude/article/ view/9740>. Acesso em: 01 mai. 2016.

THE SUPPORT. Principal investigators. A controlled trial to improve care for seriously ill hospitalized patients: the study to understand prognoses and preferences for outcomes and risk of treatment. JAMA, v. 274, p. 1591-8, 1995. 
WORLD HEALTH ORGANIZATION. Heath promotion. The Otawa Charter for. 1986. Disponivel em: <http://www.who.int/healthpromotion/ conferences/previous/ottawa/en/index4.htmls. Acesso em: 19 jun. 2018.

Cancer: palliative care. 2002. Disponivel em: <http://www.who.int/cancer/palliative/en/>. Acesso em: 19 jun. 2018.
Palliative care. 2017. Disponível em: <http://www.who.int/en/news-room/fact-sheets/ detail/palliative-care>. Acesso em: 19 jun. 2018. SAITO, D.Y.T;;ZOBOLI, E. L. C.P.Cuidados paliativos e a atenção primária à saúde: scoping review. Rev. Bioética, v. 23, n. 3, Brasília, set./dez. 2015. Disponível em: <http://www.scielo.br/scielo.php?script=sci_ arttext\&pid $=$ S1983-80422015000300593>. Acesso em: 14 mar. 2018. 
